\title{
Association of intracranial aneurysms and meningiomas: a case-control study
}

\author{
Young-Hoon Kim, MD, ${ }^{1,2}$ Young Jin Lee, MD, ${ }^{3}$ Jung Ho Han, MD, ${ }^{1,2}$ Soyeon Ahn, PhD, ${ }^{4}$ \\ Jaebong Lee, PhD, ${ }^{4}$ Jae Hyoung Kim, MD, ${ }^{5}$ Byung Se Choi, MD, ${ }^{5}$ Jae Seung Bang, MD, ${ }^{1,2}$ \\ Chae-Yong Kim, MD, ${ }^{1,2}$ Gyojun Hwang, MD, ${ }^{1}$ O-Ki Kwon, MD, ${ }^{1,2}$ and Chang Wan Oh, MD ${ }^{1,2}$
}

'Department of Neurosurgery, Seoul National University Bundang Hospital, Gyeonggi-do, Korea; '2Department of Neurosurgery, Seoul National University College of Medicine, Seoul, Korea; ${ }^{3}$ Cerebrovascular Center, Pohang Stroke and Spine Hospital, Pohang, Korea; ${ }^{4}$ Medical Research Collaborating Center and ${ }^{5}$ Department of Radiology, Seoul National University Bundang Hospital, Gyeonggi-do, Korea

\begin{abstract}
OBJECT The authors aimed to assess whether the prevalence of intracranial aneurysms in patients with intracranial meningiomas was higher than that in a healthy population.

METHODS The authors performed a hospital-based case-control study of 300 patients with newly diagnosed intracranial meningiomas and 900 age- and sex-matched controls without a history of brain tumors to evaluate any associations between intracranial aneurysms and intracranial meningiomas. Unconditional multivariate logistic regression models were used for case-control comparisons.

RESULTS Intracranial aneurysms were identified in 23 patients (7.7\%) and 24 controls (2.7\%; $p<0.001)$. There was a significant association between intracranial aneurysms and intracranial meningiomas (OR 2.913, 95\% Cl 1.613-5.261) and hypertension (OR 1.905, 95\% Cl 1.053-3.446). In a subgroup analysis of the patients with newly diagnosed intracranial meningiomas, there was a significant association between intracranial aneurysms and hypertension (OR 2.876, 95\% $\mathrm{Cl} 1.125-7.352)$ and tumor volume (OR 1.012, 95\% Cl 1.001-1.024). These patients were also more likely than controls to have other intracranial vascular diseases $(p<0.001)$, such as isolated occlusion of the intracranial vessels, excluding intracranial aneurysms.
\end{abstract}

CONCLUSIONS The prevalence of intracranial aneurysms was higher in patients with intracranial meningiomas. Hypertension and tumor volume appear to be associated with the formation of intracranial aneurysms in these patients.

http://thejns.org/doi/abs/10.3171/2014.10.JNS14710

KEY WORDS intracranial aneurysm; meningioma; hypertension; tumor volume; prevalence; oncology

$\mathrm{S}$ UBARACHNOID hemorrhage due to rupture of an intracranial aneurysm is a devastating event with high case fatality and morbidity. ${ }^{11}$ Furthermore, physicians hesitate to imagine unexpected hemorrhage from intracranial aneurysms during or after the surgical treatment of brain tumors, and such cases can result in a case mortality rate of approximately $40 \% .{ }^{9}$ Unfortunately, the coexistence of intracranial aneurysms and brain tumors is not a new phenomenon; ${ }^{9}$ however, the association between the two is still under debate..$^{7,13}$

In particular, an intracranial meningioma was reported as one of the most likely tumors to facilitate the formation of an intracranial aneurysm. ${ }^{9}$ Nonetheless, no studies have investigated the prevalence of intracranial aneurysms in patients with intracranial meningiomas; in particular, none have used specific diagnostic methods for screening intra-

ABBREVIATIONS DSA = digital subtraction angiography; MRA = MR angiography.

SUBMITTED April 29, 2014. ACCEPTED October 16, 2014.

INCLUDE WHEN CITING Published online April 17, 2015; DOI: 10.3171/2014.10.JNS14710.

DISCLOSURE The authors report no conflict of interest concerning the materials or methods used in this study or the findings specified in this paper. This study was supported by Grant No. 02-2011-006 from the SNUBH Research Fund. 
cranial vascular abnormalities. Moreover, it is still unclear whether the prevalence of intracranial aneurysms in patients with intracranial meningiomas is higher than that in a healthy population after adjusting for risk factors; thus, we conducted a retrospective case-control study.

\section{Methods \\ Patient Selection}

Our case-control study was nested within a longitudinal cohort study of patients who were treated with surgery and/or stereotactic radiosurgery for newly diagnosed intracranial meningiomas at the Seoul National University Bundang Hospital in the period from 2003 through 2012. Eligible patients included those who were treated for intracranial meningiomas and had undergone a routine preoperative evaluation and/or tumor embolization using 4-vessel intraarterial digital subtraction angiography (DSA) and/or MR angiography (MRA).

The control group consisted of patients without a history of brain tumors who were observed for routine health screening tests, including MRA, between 2003 and 2012 at the same hospital. After enrollment of each meningioma case, 3 eligible controls with the same sex and age were randomly selected from the control group.

\section{Evaluation of Intracranial Aneurysms and Definitions of Variables}

Two neurosurgeons (Y.H.K. and Y.J.L.) and 2 neuroradiology specialists (J.H.K. and B.S.C.) independently reviewed all intraarterial DSA and MRA images for intracranial vascular abnormalities, such as intracranial aneurysms and/or occlusion of the distal internal cerebral arteries or proximal middle cerebral arteries, and the results for each evaluator were integrated. All reviewers were unaware of any clinical information on the subjects.

The presence of intracranial aneurysms and/or other vascular abnormalities was ultimately unanimously confirmed by the 4 aforementioned evaluators and 3 neurosurgeons (J.H.H., J.S.B., and G.H.), particularly in differentiating intracranial aneurysms from a normal variation, such as an infundibulum of the arterial branches. Images were read using a digital picture archiving and communication system (PACS). Control patients who had been treated for intracranial aneurysms at any time before their general health screening tests were regarded as having a positive finding for intracranial aneurysms.

Information about intracranial aneurysms was gathered, including lesion size (defined as the maximum width in the dome or neck), location, and treatment. A posterior communicating artery aneurysm was classified as one in which the internal carotid artery was involved. Intracranial meningioma size (volume) was measured on MR images using the approximate volume calculation of an ellipsoid, as described elsewhere. ${ }^{5}$ Tumor location was defined as the right or left hemisphere or midline, according to the location of the tumor epicenter. The association of intracranial aneurysms with intracranial meningiomas was classified as ipsilateral, contralateral, or midline. The midline was defined as a situation in which meningiomas and/ or aneurysms were located in the midline, as in olfactory groove meningiomas and anterior communicating artery aneurysms.

Clinical data, such as patient age, sex, hypertension, tumor pathology in surgical cases, and a family history of stroke with at least one affected first-degree relative, were also collected. All medical data were based on information contained in hospital charts and radiological studies and were collected in accordance with the case record form approved by the institutional review board.

\section{Statistical Analysis}

Appropriate bivariate tests (t-test for continuous data and chi-square test for categorical variables) were performed. Unconditional multivariate logistic regression models were used to estimate odds ratios and the associated $95 \%$ confidence intervals. A p value $<0.05$ for associations was considered to indicate statistical significance. All statistical analyses were performed using the IBM SPSS Statistics package (version 20.0.0, IBM Corp.).

\section{Results}

We enrolled 404 consecutive patients who were treated for newly diagnosed intracranial meningiomas in the longitudinal cohort study for the period from 2003 through 2012, and 309 patients were identified as having pretreatment DSA or MRA results. We then performed random 1:3 case-control matching for sex and age from a database of 9557 consecutive controls. Finally, 300 patients (74.3\%) with intracranial meningiomas and 900 controls were included in this study after excluding 9 patients with intracranial meningiomas because of random case-control matching. We included 43 patients who were treated with stereotactic radiosurgery and underwent MRA before treatment for meningiomas to adjust for the effect of tumor size. Among the 300 patients with meningiomas, $55(18.3 \%)$ had MRA studies and $245(81.7 \%)$ had DSA studies. In the univariate analysis, both meningioma and control patients were similar with regard to sex, age, and family history of stroke, but those with meningioma were more likely to have hypertension than controls (Table 1).

\section{Association of Intracranial Aneurysms With Meningiomas}

Intracranial aneurysms were identified in 23 meningioma patients $(7.7 \% ; 17$ patients with single aneurysms and 6 with multiple aneurysms) and 24 controls (2.7\%; p $<0.001$ ). Among the 300 meningioma patients, the sensitivity of MRA was $5.4 \%$ (3 of 55 patients), while that of DSA was $8.2 \%$ (20 of 245). The difference in sensitivity between the 2 examination methods was not significant $(p=0.776)$. Multiple aneurysms were found in only 6 meningioma patients $(2.0 \%)$. The most common intracranial aneurysm location was the internal carotid artery in both groups. Most intracranial aneurysms were smaller than 5 $\mathrm{mm}$, and the mean aneurysm size was $4.92 \pm 4.35 \mathrm{~mm}$ (range 2.0-23.5 mm) in meningioma patients and $2.97 \pm$ $1.25 \mathrm{~mm}$ (range $1.5-6.0 \mathrm{~mm}$ ) in controls after excluding 3 patients who had undergone surgery for intracranial aneurysms before routine health screening tests. The characteristics of the patients and intracranial vascular abnormalities are summarized in Tables 1 and 2.

The unconditional multivariate analysis showed a sig- 
TABLE 1. Summary of characteristics in patients with intracranial meningiomas and age- and sex-matched controls

\begin{tabular}{|c|c|c|c|}
\hline \multirow[b]{2}{*}{ Parameter } & \multicolumn{2}{|c|}{ No. $(\%)$} & \multirow[b]{2}{*}{$p$ Value } \\
\hline & $\begin{array}{c}\text { Patients w/ } \\
\text { Meningiomas }\end{array}$ & Controls & \\
\hline Total & 300 & 900 & \\
\hline \multicolumn{4}{|l|}{ Sex } \\
\hline Female & $215(71.7)$ & $645(71.7)$ & 1.000 \\
\hline Male & $85(28.3)$ & $255(28.3)$ & \\
\hline \multicolumn{4}{|l|}{ Age in yrs } \\
\hline Mean \pm SD & $57.3 \pm 11.7$ & $57.3 \pm 11.7$ & 1.000 \\
\hline Range & $26-82$ & $26-82$ & \\
\hline \multicolumn{4}{|l|}{ Tumor vol in $\mathrm{cm}^{3}$} \\
\hline Mean \pm SD & $31.0 \pm 34.3$ & - & \\
\hline Range & $0.1-188.5$ & - & \\
\hline \multicolumn{4}{|l|}{ Treatment for meningioma } \\
\hline Surgery & $257(85.7)$ & - & \\
\hline Stereotactic radiosurgery & $43(14.3)$ & - & \\
\hline Hypertension & $108(36.0)$ & $259(28.8)$ & 0.019 \\
\hline Family history of stroke & $38(12.7)$ & $140(15.6)$ & 0.260 \\
\hline Intracranial aneurysms & $23(7.7)$ & $24(2.7)$ & $<0.001$ \\
\hline Single & $17(5.7)$ & $24(2.7)$ & \\
\hline Multiple & $6(2.0)$ & 0 & \\
\hline Other vascular disease* & $10(3.3)$ & $4(0.4)$ & $<0.001$ \\
\hline Moyamoya disease & $3(1.0)$ & 0 & \\
\hline Isolated occlusion & $5(1.7)$ & $4(0.4)$ & \\
\hline Dural AVF & $2(0.7 \%)$ & 0 & \\
\hline
\end{tabular}

$\mathrm{AVF}=$ arteriovenous fistula; $\mathrm{SD}=$ standard deviation; $-=$ not applicable

* Other vascular disease denotes intracranial vascular abnormalities of intracranial vessels, excluding intracranial aneurysms.

nificantly increased association between intracranial aneurysms and intracranial meningiomas (OR 2.913,95\% CI 1.613-5.261) and a markedly elevated association between intracranial aneurysms and hypertension (OR 1.905, 95\% CI 1.053-3.446). In the subgroup analysis of meningioma patients, there was a significant association between intracranial aneurysms and hypertension (OR 2.876, 95\% CI $1.125-7.352)$ and tumor volume (OR 1.012, 95\% CI 1.0011.024). The results of the statistical analyses are summarized in Table 3.

\section{Association of Intracranial Vascular Abnormalities With Meningiomas}

Meningioma patients were also more likely to have other intracranial vascular diseases, such as isolated occlusion of the intracranial vessels, excluding intracranial aneurysms, compared with controls $(\mathrm{p}<0.001$; Table 1$)$. Intracranial vascular abnormalities were significantly associated with intracranial meningiomas (OR 3.689, 95\% CI 2.179-6.246) and hypertension (OR 2.261, 95\% CI 1.336-3.826; Table 3). In the subgroup analysis of meningioma patients, there was a significant association between intracranial vascular abnormalities and hypertension (OR 3.248 , 95\% CI 1.449-7.277) and tumor volume (OR 1.011, 95\% CI 1.001-1.021).
TABLE 2. Characteristics of intracranial aneurysms in patients with intracranial meningioma and age- and sex-matched controls

\begin{tabular}{lcc}
\hline \multicolumn{1}{c}{ Parameter } & $\begin{array}{c}\text { Patients w/ } \\
\text { Meningiomas }\end{array}$ & Controls \\
\hline No. & 29 & 24 \\
\hline Size in mm & & \\
\hline$<5$ & 19 & 19 \\
\hline $5-9$ & 9 & 5 \\
\hline$\geq 10$ & 1 & 0 \\
\hline Location & & \\
\hline Internal carotid artery & 19 & 19 \\
\hline Anterior cerebral artery & 5 & 4 \\
\hline Middle cerebral artery & 5 & 1 \\
\hline Vertebrobasilar artery & 0 & 0 \\
\hline Association w/ intracranial meningioma & & \\
\hline Ipsilat & 13 & - \\
\hline Midline & 7 & - \\
\hline Contralat & 9 & - \\
\hline
\end{tabular}

\section{Discussion}

The prevalence of associated occurrences of intracranial aneurysms and brain tumors was first reported as < $0.3 \%$ in a series of 23,876 brain tumor patients. ${ }^{9}$ Recently, advanced techniques for imaging intracranial vessels have become increasingly available and applied, which has coincided with an increase in the incidental detection of intracranial aneurysms. ${ }^{13}$ Similarly, the recently reported prevalence of intracranial aneurysms has also increased to approximately $2 \%-5 \%$ in patients with brain tumors. ${ }^{7,8}$ Specifically, the noninvasive MRA method has been widely used to screen for intracranial vascular abnormalities; furthermore, MRA has power similar to intraarterial DSA in detecting intracranial aneurysms, as there was no significant difference in prevalence in studies with MRA as the initial imaging study compared with DSA as the reference group. ${ }^{13}$ Furthermore, the difference in sensitivity between MRA and DSA was not significant in the present study.

In addition to the recently advanced microsurgical and endovascular techniques in cases with both pathologies, the advancement of these diagnostic tools for intracranial aneurysms has decreased periprocedural mortality. ${ }^{2}$ Until now, however, the prevalence of intracranial aneurysms has not been investigated in patients with intracranial meningiomas despite the proposed possible mechanisms, such as local hemodynamic factors, hormonal influence, genetic factors, and direct invasion of tumor cells to the vessel wall.

This study provides the first evidence of an association between intracranial aneurysms and intracranial meningiomas. Although a cause-and-effect relationship cannot be inferred from a single study, our findings showed that an increased tumor volume appears to have an effect on the formation of intracranial aneurysms and other vascular abnormalities. To produce a constant cerebral perfusion pressure, arterial blood pressure should be increased by an amount equal to increases in intracranial pressure 
TABLE 3. Association of intracranial aneurysms and intracranial vascular abnormalities with risk factors

\begin{tabular}{|c|c|c|c|}
\hline Parameter & OR & $95 \% \mathrm{Cl}$ & p Value \\
\hline \multicolumn{4}{|l|}{ Intracranial aneurysms in all patients } \\
\hline Hypertension & 1.905 & $1.053-3.446$ & 0.033 \\
\hline Family history of stroke & 1.207 & $0.549-2.654$ & 0.639 \\
\hline Meningioma & 2.913 & $1.613-5.261$ & $<0.001$ \\
\hline \multicolumn{4}{|c|}{ Intracranial vascular abnormalities, including intracranial aneurysms, in all patients } \\
\hline Hypertension & 2.261 & $1.336-3.826$ & 0.002 \\
\hline Family history of stroke & 1.313 & $0.659-2.616$ & 0.438 \\
\hline Meningioma & 3.689 & $2.179-6.246$ & $<0.001$ \\
\hline \multicolumn{4}{|l|}{ Intracranial aneurysms in meningioma patients } \\
\hline Female & 1.767 & $0.615-5.078$ & 0.290 \\
\hline Age & 1.010 & $0.970-1.052$ & 0.634 \\
\hline Hypertension & 2.876 & $1.125-7.352$ & 0.027 \\
\hline Family history of stroke & 0.580 & $0.128-1.024$ & 0.479 \\
\hline Tumor vol & 1.012 & $0.001-1.024$ & 0.038 \\
\hline \multicolumn{4}{|c|}{ Intracranial vascular abnormalities, including intracranial aneurysms, in meningioma patients } \\
\hline Female & 1.857 & $0.750-4.954$ & 0.181 \\
\hline Age & 1.000 & $0.965-1.035$ & 0.988 \\
\hline Hypertension & 3.248 & $1.449-7.277$ & 0.004 \\
\hline Family history of stroke & 1.167 & $0.410-3.320$ & 0.772 \\
\hline Tumor vol & 1.011 & $1.001-1.021$ & 0.034 \\
\hline
\end{tabular}

caused by the tumor volume, even within the range of autoregulation. 1,3 Additionally, the chronic increase in arterial blood pressure may lead to an increase in hemodynamic stress, one of the principal factors of various degenerative changes in intracranial aneurysm formation, ${ }^{6}$ on cerebral arteries around slowly growing meningiomas or in the entire brain, even though it would not cause definite systemic hypertension. This long-standing hemodynamic stress also leads to the formation of intracranial aneurysms in the contralateral hemisphere and the increased prevalence of other intracranial vascular diseases, such as isolated occlusion of intracranial arteries, in patients with meningiomas.

An increase in directional blood flow in response to a higher blood supply to meningiomas was suggested as a possible source of hemodynamic stress on cerebral arterial walls. ${ }^{9,12}$ However, it may only be applicable for a small portion of cases with intracranial aneurysms located on a tumor-feeding artery or within a tumor ${ }^{2}$ because most meningiomas have major feeders from the meningeal arteries, which are branches of the external carotid artery.

Several limitations of the present study should be considered. First, the effect of genetic and hormonal factors was not evaluated in our analysis. And although their effects on this study appear to be minimal considering the low incidence of genetic disease ${ }^{4}$ and the sex- and agematched design of the present analysis, those factors may still have some effect on the co-occurrence of both pathologies in cases with relatively small tumor volumes. Furthermore, the genetic aspects of the patients and the natural history of the lesions must be considered in the studies that follow. Second, hospitalization bias may have influenced this study, particularly as regards tumor vol- ume, although we included patients treated with stereotactic radiosurgery to adjust for the effect of tumor size. However, this finding seems to be more informative to physicians responsible for patients with meningiomas, which should be treated because of their sizes. Therefore, a noninvasive vascular evaluation using MRA should be considered in selected patients to prevent an imponderable situation of intraoperative bleeding from intracranial aneurysms during surgery for brain tumors. Therefore, we cautiously recommend that intracranial meningioma patients with larger tumors or hypertension should be evaluated angiographically. Third, because the incidence of intracranial aneurysms varies from $0.5 \%$ to $7 \%$ in the general population, according to methods used to evaluate intracranial aneurysms, ${ }^{10}$ the sample size of the present study seems to be modest for determining the incidence of intracranial aneurysms in the general population. However, the prevalence of intracranial aneurysms in the healthy controls in the present study is comparable with that in another study on persons in South Korea using a similar evaluation method, 7 considering our additional adjustment for sex. In addition, the prevalence of intracranial aneurysms in this study is also comparable with those in other countries..$^{13}$ Nevertheless, our subgroup analysis of patients with intracranial meningiomas was based on smaller numbers of patients and should be interpreted with caution considering the wide confidence intervals of the analyzed variables.

\section{Conclusions}

The prevalence of intracranial aneurysms is higher in patients with intracranial meningiomas. Hypertension and 
tumor volume appear to be associated with the formation of intracranial aneurysms in these patients.

\section{References}

1. Brady KM, Lee JK, Kibler KK, Easley RB, Koehler RC, Czosnyka M, et al: The lower limit of cerebral blood flow autoregulation is increased with elevated intracranial pressure. Anesth Analg 108:1278-1283, 2009

2. Fischer BR, Palkovic S, Holling M, Niederstadt T, Jeibmann A, Wassmann H: Coexistence of cerebral aneurysm and meningioma-pure accident? Clin Neurol Neurosurg 111:647-654, 2009

3. Lassen NA: Autoregulation of cerebral blood flow. Circ Res 15 (Suppl):201-204, 1964

4. Lee JG, Lee KB, Kim UK, Ahn C, Hwang DY, Hwang YH, et al: Genetic heterogeneity in Korean families with autosomal-dominant polycystic kidney disease (ADPKD): the first Asian report. Clin Genet 60:138-144, 2001

5. Lee SH, Oh CW, Han JH, Kim CY, Kwon OK, Son YJ, et al: The effect of brain atrophy on outcome after a large cerebral infarction. J Neurol Neurosurg Psychiatry 81:1316-1321, 2010

6. Morimoto M, Miyamoto S, Mizoguchi A, Kume N, Kita T, Hashimoto N: Mouse model of cerebral aneurysm: experimental induction by renal hypertension and local hemodynamic changes. Stroke 33:1911-1915, 2002

7. Oh MC, Kim EH, Kim SH: Coexistence of intracranial aneurysm in 800 patients with surgically confirmed pituitary adenoma. J Neurosurg 116:942-947, 2012

8. Pant B, Arita K, Kurisu K, Tominaga A, Eguchi K, Uozumi $\mathrm{T}$ : Incidence of intracranial aneurysm associated with pituitary adenoma. Neurosurg Rev 20:13-17, 1997

9. Pia HW, Obrador S, Martin JG: Association of brain tumours and arterial intracranial aneurysms. Acta Neurochir (Wien) 27:189-204, 1972

10. Rinkel GJ, Djibuti M, Algra A, van Gijn J: Prevalence and risk of rupture of intracranial aneurysms: a systematic review. Stroke 29:251-256, 1998

11. Schievink WI: Intracranial aneurysms. N Engl J Med 336:28-40, 1997

12. Tachikawa T, Adachi J, Nishikawa R, Matsutani M: An anterior ethmoidal artery aneurysm associated with an olfactory groove meningioma. Case illustration. J Neurosurg 97:1479, 2002

13. Vlak MH, Algra A, Brandenburg R, Rinkel GJ: Prevalence of unruptured intracranial aneurysms, with emphasis on sex, age, comorbidity, country, and time period: a systematic review and meta-analysis. Lancet Neurol 10:626-636, 2011

\section{Author Contributions}

Conception and design: all authors. Acquisition of data: Han, YH Kim, YJ Lee, JH Kim, Choi. Analysis and interpretation of data: Han, YH Kim, YJ Lee, JH Kim, Choi. Drafting the article: Han, YH Kim, YJ Lee. Critically revising the article: Han, YH Kim. Reviewed submitted version of manuscript: Han, YH Kim. Approved the final version of the manuscript on behalf of all authors: Han. Statistical analysis: YJ Lee, Ahn, J Lee. Administrative/technical/material support: Han. Study supervision: Han, Bang, CY Kim, Hwang, Kwon, Oh.

\section{Correspondence}

Jung Ho Han, Department of Neurosurgery, Seoul National University Bundang Hospital, 82, Gumi-ro 173 Beon-gil, Bundang-gu, Seongnam-si, Gyeonggi-do 463-707, Korea. email: nstaus29@daum.net. 